\title{
PNEUMOPERITÔNIO COM DIÓXIDO DE CARBONO E IMUNOSSUPRESSÃO PÓS-OPERATÓRIA: REVISÃO SISTEMÁTICA
}

Maria Beatriz Maia Rodrigues ${ }^{1}$, Natassia Vianna Bocchese ${ }^{1}$, Noele Gurgel D'Ávila', Rebecca Renata Lapenda do Monte', Irami Araújo Filho².

1. Discente do Curso de Medicina da Universidade Potiguar, Natal RN.

2. Professor, Cirurgião do Aparelho Digestivo no Hospital Universitário Onofre Lopes e na Universidade Potiguar, Natal RN.

Study performed at Potiguar University (UNP)

Financial support: None.

Conflict of interest: None.

Correspondence address - E-mail: rebeccalapenda3@gmail.com

Submitted: april 16; accepted after revision, april 18, 2020.

\section{RESUMO}

Objetivo: O trauma cirúrgico produz disfunção imunológica profunda. As estratégias terapêuticas direcionadas à restauração da homeostase imune devem ter como objetivo corrigir o desequilíbrio fisiológico pró-inflamatório e anti-inflamatório associado a cirurgias de grande porte. Cada vez mais, tem sido comprovado a influência das cirurgias laparoscópicas para a redução da imunossupressão. O presente estudo objetivou correlacionar o uso de dióxido de carbono (CO2) em cirurgias videolaparoscópicas e a resposta imunológica pós-operatória. Métodos: Foi realizada uma revisão de literatura sistemática, através das bases de dados: PubMed/MedLine, Scopus, Embase e Web of Science and Google Scholar sobre o tema da revisão, utilizando as palavras chaves relacionadas. Os artigos foram divididos em cinco categorias: 1) Pneumoperitônio; 2) Pneumoperitônio e oncologia; 3) Cirurgia laparoscópica. Resultados: Foi observado que o trauma cirúrgico causa a liberação de citocinas inflamatórias, principalmente TNF-alfa, IL-6 e PCR. No entanto, em relação à cirurgia aberta, a laparoscopia favorece melhor imunoproteção e menores taxas de complicações pós-operatórias. Esta é a técnica preferida na maioria dos serviços de cirurgia, incluindo a cirurgia oncológica. Conclusão: A cirurgia laparoscópica é preferível à cirurgia aberta por resultar em menor comprometimento das respostas imunes e inflamatórias, visto que é menos invasiva.

Palavras-chaves: Resposta imune; Laparoscopia; Laparotomia; Dióxido de Carbono 


\section{ABSTRACT}

Purpose: Surgical trauma leads to an intense immunologic response, producing an acute systemic dysfunction. Therapeutic strategies should have the purpose to control and reclaim the fisiologic proinflammatory imbalance, produced due to large surgical interventions. In an increasingly mode, it has been proven that laparoscopic surgeries reduce the risks of postoperative immunosuppression. This present study aimed to correlate the use of carbon dioxide (CO2) in laparoscopic surgeries and to attend the immunologic immune response after the surgery. Methods: A systematic review was conducted through the databases: PubMed/MedLine, Scopus, Embase, Web of Science and Google Scholar about this review theme, using the related keywords. The articles were divided into five categories: 1) Pneumoperitoneum; 2) Pneumoperitoneum and oncology; 3) Laparoscopic surgery. Results: It has been seen that surgical trauma leads to an acute release of inflammatory cytokines, specially TNF-alpha, IL-6 and PCR. However, comparing to open surgery, laparoscopic technique promotes a better immunoprotection and lower rates of postoperative complications. Because of that, this is the first option among majority of surgical services, including oncologic surgery. Conclusion: Laparoscopic method is prefered to open surgery because of it lower commitment in immune and inflammatory responses, since it is less invasive.

Keywords: Immune System; Laparoscopy; Laparotomy; Carbon Dioxide.

\section{INTRODUÇÃO}

A reação inicial do peritônio exposto a um agente físico, químico ou bacteriano é manifestada por hiperemia, exsudação de líquido intersticial e grande influxo de células fagocitárias, principalmente macrófagos e neutrófilos. Essa resposta inflamatória é mediada por citocinas, liberadas pelos macrófagos peritoneais e pelas células mesoteliais presentes no peritônio, cuja intensidade é dependente do agente agressor envolvido ${ }^{1,2}$.

Desde 1984, existem estudos sendo elaborados para comprar os procedimentos laparoscópicos e às laparotomias. Observou-se uma redução na resposta inflamatória e imunológica sistêmica, com uma menor ativação dos mediadores químicos, representados pelas citocinas, uma menor interferência na função dos neutrófilos, em termos quantitativos e qualitativos e na integridade e ativação dos linfócitos $T^{1}$.

O trauma cirúrgico induz a um desequilíbrio metabólico, hemodinâmico, inflamatório e nutricional, levando a um estado de imunossupressão relativo, ao desencadear cascata de respostas neuroendócrinas com liberação de citocinas de fase aguda. A extensão e a duração das respostas inflamatórias e imunológicas no pósoperatório são proporcionais à magnitude do trauma cirúrgico. Quanto maior a operação maior será a resposta inflamatória. Neste aspecto, a cirurgia do trauma 
Pneumoperitônio com dióxido de carbono e imunossupressão pós-operatória

Rodrigues $M B M$, et al

(urgência/emergência) e as cirurgias oncológicas com grandes ressecções se enquadram perfeitamente ${ }^{2}$.

A imunossupressão está relacionada a complicações infecciosas, além de dor, complicações cardiopulmonares, tromboembólicas, paralisia gastrointestinal e convalescença prolongada. Assim, as técnicas operatórias que visam reduzir o impacto do trauma cirúrgico são constantemente analisadas, visando obter uma menor resposta endócrina-metabólica ${ }^{3}$.

As citocinas são aceitas como marcadores de trauma tecidual e resposta imune do hospedeiro no pós-operatório. O fator de necrose tumoral alfa (TNF-a), a interleucina 1b (IL-1b), interleucinas 6 e 8 (IL-6; IL-8) e a proteína-C-reativa (PCR) são os principais mediadores de resposta aguda em humanos ${ }^{1,2}$.

Nesse contexto, as intervenções laparoscópicas, apesar do aumento de cloreto e lactato, e redução do pH peritoneal, possibilitam uma menor morbidade pósoperatória, ao reduzir o estresse provocado pelo ato cirúrgico, em virtude do menor trauma tecidual provocado, a começar pelo acesso às cavidades corporais ${ }^{1}$.

Neste sentido, o objetivo do estudo foi analisar o impacto imunológico, inflamatório e metabólico em cirurgias que utilizam o CO2 para insuflação da cavidade peritoneal, além de comparar o pneumoperitônio com $\mathrm{CO} 2$, com outros gases e pela via aberta na cirurgia oncológica.

\section{MÉTODOS}

Realizou-se pesquisa nas bases de dados Pubmed, Scopus, Scielo, Embase e Web of Science além da busca no Google Scholar, considerado como fonte de literatura cinza, por não conter artigos revisados por pares. Foram selecionados artigos relacionados ao uso de dióxido de carbono ( $\mathrm{CO} 2$ ) em cirurgias videolaparoscópicas e a resposta imunológica pós-operatória nos últimos cinco anos, sendo escolhidos através da combinação das seguintes palavras chaves: laparoscopy and laparotomy and cancer surgery and immunosuppression. Incluiu-se todos os estudos relevantes publicados nas modalidades coorte, revisão sistemática, caso controle, transversal, estudos de séries de casos e ensaios clínicos randomizados. Utilizou-se as escalas de Jadad e NewcastleOttawa para classificação qualitativa dos principais artigos sobre o assunto. Os filtros escolhidos foram estudos realizados em humanos, artigos completos e publicados nos últimos cinco anos totalizando vinte e quatro artigos. A análise, revisão e seleção dos artigos foi feita por pares, separadamente, a partir da leitura do título e resumo do estudo, havendo um terceiro revisor em caso de discordância entre os outros dois revisores. 
Pneumoperitônio com dióxido de carbono e imunossupressão pós-operatória

Rodrigues $M B M$, et al

\section{RESULTADOS E DISCUSSÃO}

\section{Pneumoperitônio}

O pneumoperitônio, na cirurgia laparoscópica, é realizada com agentes químicos gasosos, como dióxido de carbono, óxido nitroso (N2O), argônio e hélio (He). O CO2 é atualmente o mais utilizado, por ser um gás não inflamável e por apresentar alto grau de difusão, favorecendo sua eliminação. Ainda possui como vantagem, o seu baixo custo e reduzido efeitos colaterais. Entre as desvantagens do uso desse gás estão a redução do débito cardíaco e o incremento no número de possíveis acidentes vasculares encefálicos, como consequência do aumento da pressão abdominal ${ }^{4}$.

A cavidade abdominal é revestida por peritônio com múltiplas funções, como regulação dos processos de inflamação, fibrinólise, angiogênese e remodelação de tecidos. As alterações celulares do peritônio modificam-se de acordo com o gás escolhido, com a quantidade de pressão e com a duração de insuflação. Assim, foi descrito que o pneumoperitônio por $\mathrm{CO} 2$ ou He, apresenta alterações intercelulares mais prejudiciais do que ao comparar com ar normal. Esses gases desregulam funções essenciais, como a produção de energia (ATP), a proliferação celular e o início da apoptose. No entanto, foi visto que a insuflação com $\mathrm{CO} 2$ aquecido e úmido resultou em menores alterações teciduais do que frio e seco ${ }^{1,2}$.

Em relação a resposta imunológica, notou-se que a insuflação com $\mathrm{CO} 2$ induziu a uma menor liberação de IL-1 e TNF-alfa pelos macrófagos, em comparação ao gás hélio e ao ar, ou seja, o CO2 induz a uma menor resposta inflamatória. O mecanismo exato ainda é desconhecido, mas acredita-se que este gás ao diminuir o $\mathrm{pH}$ intracelular provoca uma diminuição na produção de citocinas ${ }^{5}$.

Ao comparar as mudanças nos níveis de células natural killer (NK) e linfócitos T (LT) em material peritoneal após um pneumoperitônio induzido por $\mathrm{CO} 2$ e uma laparotomia, concluiu-se que a resposta imune mediada por células da cavidade peritoneal foi menos comprometida com o $\mathrm{CO}^{6}$.

Apesar do aumento dos LT com o CO2 ser semelhante ao da cirurgia aberta, a relação CD4/CD8 no 3o dia de pós-operatório foi menor com o dióxido de carbono, assim como o incremento de células NK. Além disso, a laparotomia mostrou-se uma forte estimuladora de secreção de citocinas inflamatórias (IL-8, IL-1 e TNF-alfa), gerando uma resposta inflamatória mais predominante ao trauma cirúrgico ${ }^{6}$.

A depuração peritoneal de bactérias também revelou-se mais eficaz após a insuflação com $\mathrm{CO} 2$ do que por via aberta. Na laparotomia foi descrita uma menor depuração das bactérias Escherichia coli, relacionada a níveis de IL-6 e TNF alfa predominantemente mais elevados no líquido peritoneal, contribuindo assim com a evolução para a sepse com níveis menores de bacteremia ${ }^{4}$.

Dessa maneira, o pneumoperitônio por $\mathrm{CO} 2$ é mostrado como benéfico em relação aos outros gases e a cirurgia aberta, ao gerar uma resposta inflamatória de 
Pneumoperitônio com dióxido de carbono e imunossupressão pós-operatória

Rodrigues $M B M$, et al

menor intensidade, permitindo uma recuperação no pós-operatório de maneira mais eficaz e precoce.

\section{Pneumoperitônio e oncologia}

Com o desenvolvimento das técnicas cirúrgicas videolaparoscópicas, o pneumoperitônio utilizando $\mathrm{CO} 2$ passou a ser utilizado em pacientes portadores de patologias cirúrgicas benignas e malignas. A utilização desse método em cirurgia oncológica é controversa pela suspeita de atuar na difusão de células tumorais, como por exemplo, por meio da manipulação com instrumentos que entram em contato direto, participando da disseminação e podendo estar na gênese da carcinomatose peritoneal. Contudo, esse tema ainda é controverso e fonte de discussão entre os cirurgiões mais experientes no âmbito da comunidade acadêmica?.

Veenhof AAFA et al em seu estudo randomizado comparando a videolaparoscopia com a laparotomia na excisão do mesorreto em pacientes com câncer retal mostraram satisfatórios resultado na resposta imune a curto prazo. Foram vistos níveis mais baixos de IL-6, duas horas após a cirurgia, assim como, uma expressão de HLA-DR mais preservada, demonstrando um combate mais significativo a infecção e a disseminação de células tumorais, e portanto, de menor risco na formação de metástases ${ }^{7}$.

No entanto, ainda não é possível concluir a origem das metástases de parede abdominal advindas, pois uma série de fatores estão envolvidos, como uso de $\mathrm{CO} 2$, efeitos citotóxicos, além da resposta imune. Fato é que o pneumoperitônio com $\mathrm{CO} 2$ causa alterações morfológicas nas células mesoteliais, porém as células tumorais só são capazes de infiltrarem e proliferarem se as mesoteliais se romperem, o que não foi comprovado na laparoscopia ${ }^{1}$.

Quando comparada a laparoscopia com a laparotomia, aquela possui uma diminuição na ativação células natural killer (NK) o que não foi detectado, por sua vez na cirurgia convencional, em que a resposta citotóxica é aumentada,resultando em maior prejuízo a nível de proliferação tumoral ${ }^{8}$.

Ademais, acredita-se que o gás carbônico insuflado também induz uma acidose peritoneal, independente do $\mathrm{pH}$ sistêmico, diante disso existe a hipótese de que essa acidificação poderia ser favorável para o implante de células tumorais no peritônio. Por outro lado, essa alteração metabólica reduz a expressão de fator de necrose tumoral alfa, sendo responsável, por uma diminuição da resposta inflamatória, que pode vir a prejudicar a implantação do tumor ${ }^{1,2}$.

A preservação da função imune no pós-operatório é importante ao permitir uma recuperação mais eficaz, podendo ser alcançada por técnicas cirúrgicas menos invasivas, como no caso da videolaparoscopia. Outro ponto favorável, é a menor manipulação dos tumores por via laparoscópica, diminuindo as chances de complicações e distribuição de células tumorais. 
Pneumoperitônio com dióxido de carbono e imunossupressão pós-operatória

Rodrigues $M B M$, et al

\section{Cirurgia laparoscópica}

Devido a menor incisão realizada nas cirurgias por via videolaparoscópicas o uso de gases para insuflar a cavidade abdominal se tornou uma necessidade. Por seu menor potencial de causar complicações pós-operatórias, o dióxido de carbono (CO2) tem sido escolhido para essa finalidade. Atualmente sabe-se que, por induzir menor resposta metabólica ao trauma, seu uso está associado a menores taxas de dor no pósoperatório, a uma recuperação precoce da função intestinal e menor imunossupressão. As taxas de infecção de ferida operatória consequentemente também apresentaram queda ${ }^{2,11,12}$. No entanto, esses benefícios são melhor observados quando utilizado o CO2 é umidificado e aquecido. O CO2 frio gera maiores chances de hipotermia no pósoperatório, além de maior chance de aderências e dor ${ }^{2,12}$.

Apesar dos inúmeros benefícios, a cirurgia por via laparoscópica, assim como qualquer tratamento, não é isenta de complicações. Sabe-se que o mínimo dano tecidual pode trazer consequências para o indivíduo, pois estudos observacionais mostraram que após poucas horas do início desse procedimento cirúrgico já se pôde observar infiltrado linfocitário e aumento na população de macrófagos. Alterações celulares e morfológicas também podem ser observadas após o uso do pneumoperitônio para insuflar a cavidade abdominal ${ }^{1,1,13}$.

A acidose local pode ocorrer por uma menor oxigenação causada pelo uso do pneumoperitônio e aumento da pressão parcial de CO2 na cavidade. Ainda, através da hipóxia transitória há uma maior propensão de formação das aderências. Todavia, esse quadro é reversível após a desinsuflação ${ }^{2,10-13}$.

Um dos outros benefícios tem sido em relação as proteínas de fase aguda, comumente aumentadas após traumas cirúrgicos e não cirúrgicos no paciente. Foi observado que estas encontravam-se em valores menores que os habituais após cirurgias videolaparoscópicas. O principal exemplo foi da proteína $C$ reativa (PCR) que geralmente permanece aumentada por cerca de duas semanas após laparotomias convencionais, mas não apresentou valores tão elevados após a videolaparoscopia, apresentando um pico durante cerca de 24-48 horas no pós-operatório. A IL-1, IL-6 e TNF-alfa também apresentaram valores menores que os habituais na laparotomia ${ }^{10,13}$.

Quanto às infecções de sítio cirúrgico é considerado fator de risco a conversão para cirurgia aberta, assim como: vazamento de anastomoses, índice de massa corporal elevado e sexo masculino. Ainda, nas cirurgias de grande porte, existe maior permeabilidade da parede intestinal predispondo a translocação bacteriana do lúmen intestinal para a cavidade peritoneal. Esse fato, contribui para uma resposta inflamatória sistêmica exacerbada, taxas elevadas de infecção de ferida operatória e sepse $^{8}$.

Dessa forma, as modalidades de cirurgias abertas mostraram-se com uma maior resposta imunodepressora, contribuindo para um maior risco de sepse, recuperação tardia e níveis elevados de morbimortalidade ${ }^{8}$. 
Pneumoperitônio com dióxido de carbono e imunossupressão pós-operatória

Rodrigues $M B M$, et al

\section{CONCLUSÃO}

Portanto, a cirurgia laparoscópica é preferível à cirurgia aberta ao resultar em menor comprometimento das respostas imunes e inflamatórias, além de ser menos invasiva. A resposta imunológica mediada por células é menos prejudicada com o pneumoperitônio. Também ocorre uma deflagração menos exuberante das citocinas inflamatórias, principalmente da IL-6, IL-1b, PCR e TNF-alfa ${ }^{14,15}$.

Um outro benefício demonstrado em literatura dos benefícios da cirurgia laparoscópica em detrimento da aberta foram: a recuperação mais rápida do paciente, menores taxas de complicações, menor tempo de íleo paralítico e mais rápida introdução de dieta ${ }^{16,17}$.

Além disso, foi detectado níveis mais baixos de complicações metabólicas, tromboembólicas, cardiopulmonares e de dor pós-operatória, consequentemente diminuindo o tempo de internação hospitalar ${ }^{18,19}$.

Apesar de ainda faltarem estudos para comprovar a adequação ou não da laparoscopia com $\mathrm{CO} 2$ em cirurgias oncológicas, sabe-se que esta técnica permite uma preservação imunológica mais eficaz no pós-operatório, sendo um ponto chave na recuperação contra o câncer. Ainda necessitamos de mais estudos para avaliar se o uso do CO2 está ou não relacionado ao aparecimento de metástases de parede abdominal, entretanto o risco está mais relacionado às condições do paciente e a cirurgia propriamente dita do que o uso do pneumoperitônio ${ }^{20,21}$.

Em cirurgias oncológicas, vários estudos já foram realizados com o intuito de buscar uma associação entre o uso do pneumoperitônio e a disseminação peritoneal de metástases. No entanto, os resultados foram inconclusivos, por esse motivo e pelos benefícios apresentados, a laparoscopia permanece sendo utilizada com esse intuito.

\section{REFERÊNCIAS}

1. Brokelman WJA, Lensvelt $M$, Rinkes IHMB, Klinkenbijl JHG, Reijnen MMPJ. Peritoneal changes due to laparoscopic surgery. Surg Endosc. 2010; 25(1):1-9.

2. Binda MM. Humidification during laparoscopic surgery: overview of the clinical benefits of using humidified gas during laparoscopic surgery. Arch Gynecol Obst. 2015; 292(5): 955-71.

3. Fondrinier E, Descamps $P$, Arnaud JP, Pezet D. [Carbon dioxide pneumoperitoneum and peritoneal carcinosis: review]. J Gynecol Obstet Biol Reprod (Paris). 2002;31(1):11-27.

4. SIDO B. Inflammatory response after abdominal surgery. Best Pract Res Clin Anaesthesiol. 2004; 18(3), 439-54. 
5. Ure BM, Niewold TA, Bax NMA, Ham M, van der Zee DC, Essen GJ. Peritoneal, systemic, and distant organ inflammatory responses are reduced by a laparoscopic approach and carbon dioxide vs air. Surg Endosc Interv Tech. 2002; 16(5): 836-42.

6. Fujii K, Izumi K, Sonoda K, Shiraishi N, Adachi Y, Kitano S. Less Impaired cellmediated immune response in the murine peritoneal cavity after $\mathrm{CO} 2$ pneumoperitoneum. Surg Today. 2003; 33(11): 833-8.

7. Veenhof AAFA, Sietses $C$, von Blomberg BME, van Hoogstraten IMW, vd Pas MHGM, Meijerink WJHJ et al. The surgical stress response and postoperative immune function after laparoscopic or conventional total mesorectal excision in rectal cancer: a randomized trial. Int J Colorectal Dis. 2010; 26(1): 53-9.

8. Ni Choileain N, Redmond HP. Cell Response to Surgery. Jama Surgery. Arch Surg. 2006 Nov;141(11):1132-40.

9. Kwak HJ, Jo YY, Lee KC, Kim YB, Shinn HK,Kim JY. Acid-base alterations during laparoscopic abdominal surgery: a comparison with laparotomy. Br J Anaesth. 2010; 105(4): 442-7.

10. De Sousa GC, Cruz FF, Heil LB, Sobrinho CJS, Saddy F, Knibel FP et al. Intraoperative immunomodulatory effects of sevoflurane versus total intravenous anesthesia with propofol in bariatric surgery (the OBESITA trial): study protocol for a randomized controlled pilot trial. Trials. 2019; 20(1):300.

11. Tuna AT, Akkoyun I, Darcin S, Palabiyik O. Effects of carbon dioxide insufflation on regional cerebral oxygenation during laparoscopic surgery in children: a prospective study. Braz J Anesthesiol. 2016; 66(3): 249-53.

12. Birch DW, Dang JT, Switzer NJ, Manouchehri N, Shi X, Hadi G, Karmali S. Heated insufflation with or without humidification for laparoscopic abdominal surgery. Cochrane Database System Reviews. 2016. doi:10.1002/14651858.cd007821.pub3

13. Mason SE, Kinross JM, Hendricks J, Arulampalam TH. Postoperative hypothermia and surgical site infection following peritoneal insufflation with warm, humidified carbon dioxide during laparoscopic colorectal surgery: a cohort study with costeffectiveness analysis. Surg Endosc. 2017;31(4):1923-9.

14. Fujii K, Izumi K, Sonoda K, Shiraishi N, Adachi Y, Kitano S. Less Impaired CellMediated Immune Response in the Murine Peritoneal Cavity After $\mathrm{CO} 2$ Pneumoperitoneum. Surgery Today. 2003; 33(11): 833-8.

15. Sammour T, Kahokehr A, Chan S, Booth RJ, Hill AG. The humoral response after laparoscopic versus open colorectal surgery: A meta-analysis. J Surg Res. 2010; 164(1): 28-37.

16. Schietroma M, Colozzi S, Pessia B, Carlei F, Di Furia M, Amicucci G. Laparoscopic Nissen fundoplication: The effects of high-concentration supplemental 
perioperative oxygen on the inflammatory and immune response: $\mathrm{A}$ randomised controlled trial. J Minim Access Surg. 2018;14(3):221-9.

17. Kuhry E, Jeekel J,Bonjer HJ. Effect of laparoscopy on the immune system. Surg Innov. 2004; 11(1): 37-44.

18. Sylla P, Kirma I, Whelan RL. Immunological advantages of advanced laparoscopy. Surg Cl North Am. 2005; 85(1): 1-18.

19. Jesch NK, Kuebler JF, Nguyen $H$, Nave $H$, Bottlaender $M$, Teichmann $B$ et al. Laparoscopy vs minilaparotomy and full laparotomy preserves circulatory but not peritoneal and pulmonary immune responses. J Ped Surg. 2006; 41(6): 1085-92.

20. Mo X, Yang Y, Lai H, Xiao J, He K, Chen J, Lin Y. Does carbon dioxide pneumoperitoneum enhance wound metastases following laparoscopic abdominal tumor surgery? A meta-analysis of 20 randomized control studies. Tumor Biol. 2014; 35(8):7351-9.

21. Watt DG, Horgan PG, McMillan DC. Routine clinical markers of the magnitude of the systemic inflammatory response after elective operation: A systematic review. Surgery. 2015;157(2): 362-80. 